$\underline{\text { Review Article }}$

\title{
NANOSUSPENSION: AN OVERVIEW
}

\author{
SMITA S. AHER ${ }^{* 1}$, SAGAR T. MALSANE ${ }^{2}$, R. B. SAUDAGAR ${ }^{1}$
}

1*Department of Pharmaceutical Chemistry, R. G. Sapkal College of Pharmacy, Anjaneri, Nashik 422213, Maharashtra, India, ${ }^{2}$ Department of Quality Assurance Techniques, R. G. Sapkal College of Pharmacy, Anjaneri, Nashik 422213, Maharashtra, India

Email: sagarmalsane14@gmail.com

Received: 27 Dec 2016, Revised and Accepted: 27 Mar 2017

\begin{abstract}
Nanosuspensions are important carriers to develop novel drug formulations. Nanosuspensions have emerged as a promising strategy for the efficient delivery of hydrophobic drugs because of their versatile features and unique advantages. Nanosuspension technology solved the problem of drugs which are poorly aqueous soluble and less bioavailability. Stability and bioavailability of the drugs can be improved by the Nanosuspension technology. Techniques such as media milling and high-pressure homogenization have been used commercially for producing nanosuspensions. Nanosuspensions can be delivered by oral, parenteral, pulmonary and ocular routes. Nanosuspensions can also be used for targeted drug delivery when incorporated in theOcular inserts and mucoadhesive hydrogels. Currently, efforts are being directed to extending their applications in sitespecific drug delivery.
\end{abstract}

Keywords: Nanosuspension, Solubility enhancement, Particle size, Surfactant, Colloidal dispersion, Oral bioavailability

(C) 2017 The Authors. Published by Innovare Academic Sciences Pvt Ltd. This is an open access article under the CC BY license (http://creativecommons.org/licenses/by/4.0/] DOI: http://dx.doi.org/10.22159/ijcpr.2017v9i3.19584

\section{INTRODUCTION}

Nanosuspension is a submicron colloidal dispersion of drug particles. A pharmaceutical nanosuspension is defined as very finely colloid [1], biphasic [2], dispersed solid drug particles in an aqueous vehicle, a size below $1 \mu \mathrm{m}$ stabilized by surfactants [3] and polymers [4] prepared by suitable methods for drug delivery [5] applications.

Nanosuspension have revealed their potential to solve the problem [6] associated with the delivery of poorly water-soluble and poorly water and lipid soluble drugs. It enhances the absorption and bioavailability and helps to reduce the dose of conventional oral dosage forms. Drug particle size reduction leads to an increase in the surface area and consequently the rate of dissolution as described by the Noyes Whitney [7] equation. In addition, an increase in saturation solubility is postulated by the particle size reduction due to an increased dissolution pressure explained by the Ostwald-Freundlich equation [8]. Depending upon the production technique applied changes in the crystalline structure of the drug particle may occur. An increasing amount of amorphous drug fraction could induce higher saturation solubility. Nanosuspension not only solves the problem of poor solubility and poor bioavailability but also alters the pharmacokinetics of the drug and improves the drug safety and efficacy [9].

\section{Criteria for selection of drug for nanosuspensions}

Nanosuspension can be prepared for the API that is having either of the following characteristics:

a) Water insoluble but which are soluble in oil b) (high $\log$ ) or API are insoluble in both water and oils

c) Drugs with reduced tendency of the crystal to dissolve, regardless of the solvent

d) API with very large dose.

\section{Advantages}

a. Most cost effective.

b. Useful for poorly soluble drugs.

c. Physically more stable than liposomes.

d. Provide ease of manufacture and scale up for large scale production.

e. Rapid dissolution and tissue targeting.

f. Reduction in tissue irritation.

g. Higher bioavailability in ocular and inhalational drug delivery

Preparation of nanosuspension

Technically preparations of nanosuspensions are a simpler alternative than liposome's and other conventional colloidal drug carriers but reported to be more cost effective. It is particularly for poorly soluble drugs and to yield a physically more stable product. For manufacturing nanosuspensions, there are two converse methods, "Top-down process technology" and "Bottom-up process technology". The top-down process follows disintegration approach from large particles, microparticles to Nanosized particles $[10,11]$.

Table 1: Advantages of nanosuspensions over conventional formulations

\begin{tabular}{lll}
\hline $\begin{array}{l}\text { Route of } \\
\text { administration }\end{array}$ & $\begin{array}{l}\text { Disadvantages of conventional } \\
\text { formulations }\end{array}$ & Benefits of nanosuspensions \\
\hline Oral & Slow onset of action/poor absorption & Rapid onset of action/improved solubility so improved bioavailability \\
Ocular & Lachrymal wash off/low bioavailability & Higher bioavailability/dose consistency \\
Intravenous & Poor dissolution/nonspecific action & Rapid dissolution/tissue targeting \\
Intramuscular & Low patient compliance due to pain & Reduced tissue irritation \\
Inhalations & Low bioavailability due to low solubility & Rapid dissolution/high bioavailability/dose regulation \\
\hline
\end{tabular}

\section{Examples are}

a) High-pressure homogenization b) Nanopure

c) Media milling (nanocrystals). 
d) Bottom-up process is an assembly method forms nanoparticles from molecules. Examples includes;

1. Solvent-antisolvent method

2. Super critical fluid process

3. Emulsification Solvent evaporation technique

4. Lipid emulsion/Micro-emulsion template.

The principle techniques used in recent years for preparing nanosuspensions are

A. High-pressure homogenization

B. Milling techniques

C. Emulsification-solvent

D. Precipitation

E. Supercritical fluid process

F. Melt emulsification method

G. Lipid emulsion/microemulsion template

H. Solvent evaporation

\section{A. High-pressure homogenization}

In the high-pressure homogenization method, the suspension of a drug and surfactant is forced under pressure through a nanosized aperture valve of a high-pressure homogenizer. The principle of this method is based on cavitation forces of drug particles in the aqueous phase. These forces are sufficiently high to convert the drug microparticles into nanoparticles.

\section{Homogenization in aqueous media (Disso. cubes)}

This technology was developed by R. H. Muller using a piston-gap type high-pressure homogenizer in 1999. In this method, the suspension containing a drug and surfactant is forced under pressure through a Nanosized aperture valve of a high-pressure homogenizer.

\section{Principle}

This method is based on cavitation principle. The dispersion present in $3 \mathrm{~cm}$ diameter cylinder is suddenly passed through a very narrow gap of $25 \mu \mathrm{m}$. According to Bernoulli's law, the flow volume of liquid in a closed system per cross section is constant. It leads to increase in dynamic pressure and decrease of static pressure below the boiling point of water at room temperature due to a reduction in diameter from $3 \mathrm{~cm}$ to $25 \mu \mathrm{m}$. Then water starts boiling at room temperature and forms gas bubbles, which implode when the suspension leaves the gap (called cavitation) and normal air pressure is reached. The particles cavitation forces are sufficiently high to convert the drug microparticles into nanoparticles.

\section{Homogenization in non-aqueous media}

i. Nanopure: Nanopure is suspensions homogenized in water-free media or water mixtures like PEG 400, PEG 1000 etc. The homogenization can be done at room temperature, $0{ }^{\circ} \mathrm{C}$ and below freezing point (-200C), hence it is known as "deep freeze" homogenization.

ii. Nanoedge: Nanoedge technology is the combination of both precipitation and homogenization. The basic principle is same as that of precipitation and homogenization. The major disadvantage of precipitation technique such as crystal growth and long-term stability can be overcome by using the Nanoedge technology. Particles of smaller size and better stability in a short time can be achieved.

iii. Nanojet: It is also called as opposite stream technology, uses a chamber where a stream of suspension is divided into two or more parts, which colloid with each other at high pressure, due to the high shear forces produced during the process particle size is reduced.

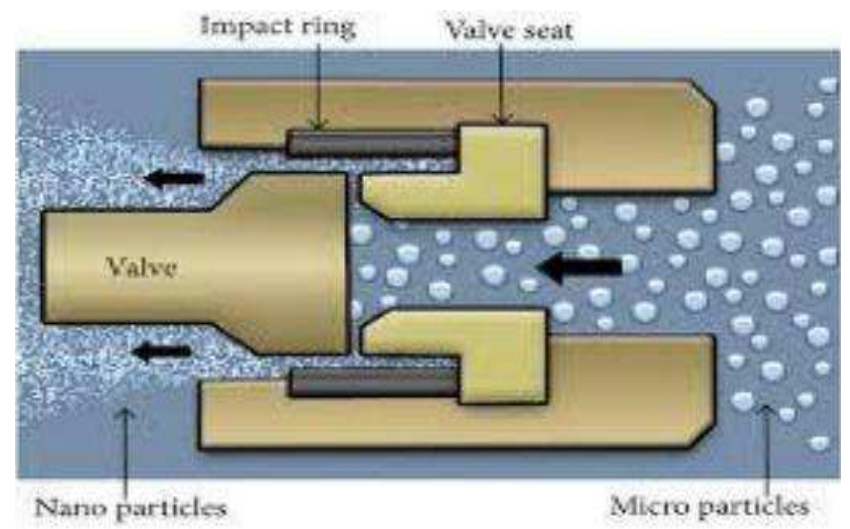

Fig. 1: Schematic cartoon of the high-pressure homogenization process

\section{Media milling}

\section{i. Media milling}

Media milling is a further technique used to prepare Nanosuspensions. Nanocrystal is a patent protected technology US 5,145,684 developed by Élan Nanosystems. In this technique, the drug nanoparticles are obtained by subjecting the drug to media milling. High energy and shear forces generated as a result of impaction of the milling media with the drug provide the necessary energy input to disintegrate the microparticulate drug into nanosized particles.

In the media milling process, the milling chamber is charged with the milling media, water or a suitable buffer, drug and stabilizer. Then the milling media or pearls are rotated at a very high shear rate.

\section{ii. Dry co-grinding}

Recently, Nanosuspensions can be obtained by dry milling techniques. Dry co-grinding can be carried out easily and economically and can be conducted without organic solvents. The co-grinding technique can reduce particles to the submicron level and a stable amorphous solid can be obtained.

\section{Emulsification-solvent}

\section{Evaporation technique}

This technique involves preparing a solution of drug followed by its emulsification in another liquid that is a nonsolvent for the drug. Evaporation of the solvent leads to precipitation of the drug. Crystal growth and particle aggregation can be controlled by creating high shear forces using a high-speed stirrer 


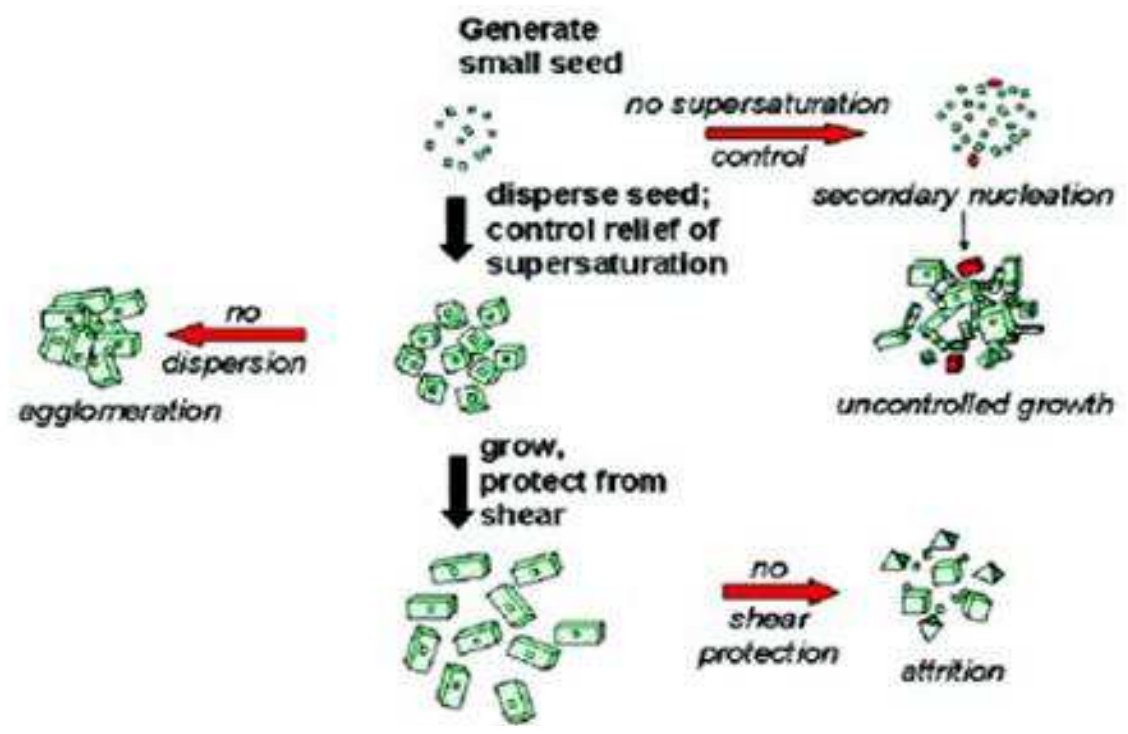

Fig. 2: Media milling process

\section{Precipitation}

\section{Precipitation technique (solvent-anti-solvent method)}

Precipitation method has been used for long years for the preparation of submicron particles. It is mainly used for the poorly soluble drugs. The first drug is dissolved in a suitable solvent. This solution is then mixed with a miscible antisolvent system in the presence of surfactants. Rapid addition of drug solution into the antisolvent leads to the sudden supersaturation of drug in the mixed solution forms ultrafine drug solids. Precipitation method involves two phases-nuclei formations and crystal growth. When preparing a stable suspension with the minimum particle size, a high nucleation rate and but the low growth rate is necessary. Both rates are depend on temperature. In this technique, the drug needs to be soluble in at least one solvent which is miscible with a nonsolvent.

\section{E. Supercritical fluid process}

Supercritical fluid technology can be used to produce nanoparticles from drug solutions. The various methods attempted are rapid expansion of supercritical solution process (RESS), supercritical anti-solvent process and precipitation with compressed anti-solvent process (PCA). The RESS involves expansion of the drug solution in supercritical fluid through a nozzle, which leads to loss of solvent power of the supercritical fluid resulting in precipitation of the drug as fine particles. Young et al. prepared cyclosporine nanoparticles in the size range of 400-700 $\mathrm{nm}$ using this process. In the PCA method, the drug solution is atomized into a chamber containing compressed CO 2. As the solvent is removed, the solution gets supersaturated and thus precipitates as fine crystals. The supercritical anti-solvent process uses a supercritical fluid in which a drug is poorly soluble and a solvent for the drug that is also miscible with the supercritical fluid. The drug solution is injected into the supercritical fluid and the solvent gets extracted by the supercritical fluid and the drug solution gets supersaturated. The drug is then precipitated as fine crystals. Nanoparticles of griseofulvin, a drug with poor solubility, using this method.

\section{F. Melt emulsification method}

In this method, the drug is dispersed in the aqueous solution of stabilizer and heated above the melting point of the drug and homogenized to give an emulsion. During this process, the sample holder was enwrapped with a heating tape fitted with a temperature controller and the temperature of the emulsion was maintained above the melting point of the drug. The emulsion was then cooled down either slowly to room temperature or on an ice-bath. The main advantage of melt emulsification technique relative to the solvent diffusion method is total avoidance of organic solvents during the production process. Nanosuspension of ibuprofen was prepared by this method. Formulating ibuprofen Nanosuspension by melt emulsification method show greater dissolution rate than formulating by the solvent diffusion method.

\section{G. Lipid emulsion/microemulsion template}

Another way to produce nanosuspensions is to use an emulsion which is formed by the conventional method using a partially water miscible solvent as the dispersed phase. Nanosuspensions are obtained by just diluting the emulsion. Moreover, microemulsions as templates can produce nanosuspensions. Microemulsions are thermodynamically stable and isotropically clear dispersions of two immiscible liquids such as oil and water stabilized by an interfacial film of surfactant and co-surfactant. The drug can be either loaded into the internal phase or the pre-formed microemulsion can be saturated with the drug by intimate mixing. Suitable dilution of the microemulsion yields the drug nanosuspension. An example of this technique is the griseofulvin nanosuspension which is prepared by the microemulsion technique using water, butyl lactate, lecithin and the sodium salt of taurodeoxycholate. The advantages of lipid emulsions as templates for nanosuspension formation are that they easy to produce by controlling the emulsion droplet and easy for scale-up. However, the use of organic solvents affects the environment and large amounts of surfactant or stabilizer are required.

\section{H. Solvent evaporation}

In the solvent evaporation method, the solutions of the polymer are prepared in volatile solvents and emulsions. The emulsion is converted into a nanoparticle suspension on evaporation of the solvent for the polymer, which is allowed to diffuse through the continuous phase of the emulsion. The particle size was influenced by the concentration of polymer, stabilizer and the speed of homogenizer. In the conventional methods, two main strategies are being used for the formation of emulsions, the preparation of single emulsions, e. g., oil-in-water (o/w) or double-emulsions, e. g., (water-in-oil)-in-water, (w/o)/w.

These methods require high-speed homogenization or ultrasonication, followed by evaporation of the solvent, either by continuous magnetic stirring at room temperature or under reduced pressure. By ultracentrifugation, the solidified nanoparticles are collected which was washed with distilled water to remove the additives like surfactants, and then it was lyophilized Single-emulsions, e. g., oil-in-water $(\mathrm{o} / \mathrm{w})$ or doubleemulsions, e. g., (water-in-oil)-in-water, (w/o)/w[12]. 


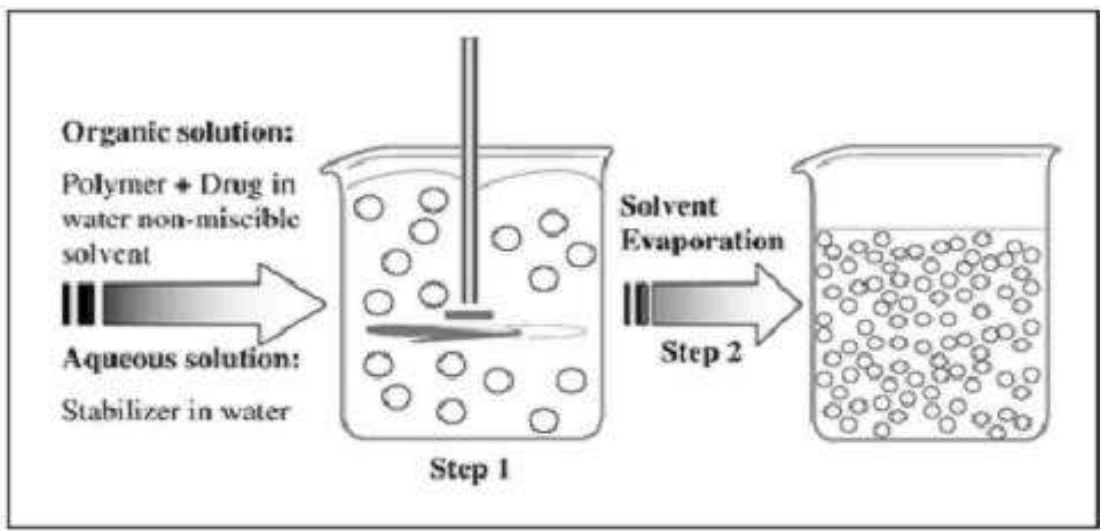

Fig. 3: Schematic representation of the solvent evaporation technique
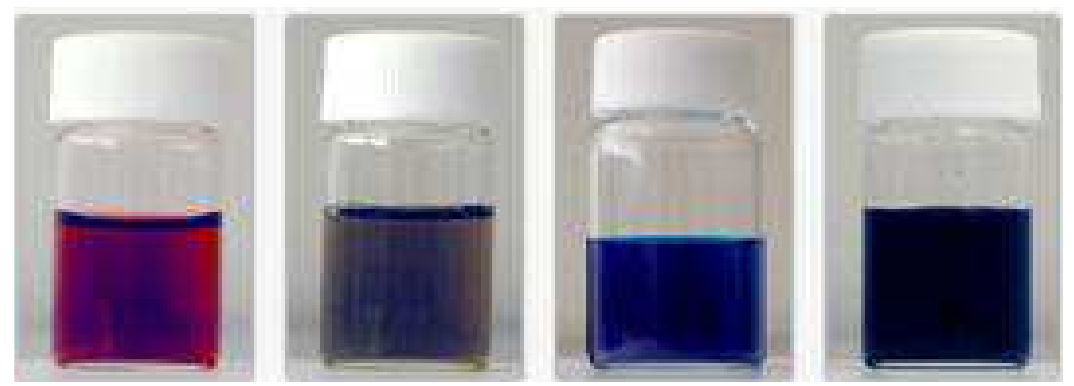

Fig. 4: Nanosuspensions for oral drug delivery

\section{Application}

Nanosuspensions have a wide range of applications especially in the case of low solubility and low bioavailability drugs. They are mentioned below.

\section{Oral drug delivery}

Because of the numerous advantages, oral route is a preferable route for many of the drugs (fig. 3) especially in the case of orally administering antibiotics such as atovaquone and buparvaquone. By making it in nano size, its solubility and bioavailability will increase. The oral administration of naproxen nanoparticles leads to an area under the curve (AUC) $(0-24 \mathrm{~h})$ of $97.5 \mathrm{mg}-\mathrm{h} / \mathrm{l}$ compared with naproxen nanosuspension and naproxen tablets 16 . In the case of danazole (gonadotropin inhibitor), nanosuspension has an absolute bioavailability of 82.3 and the conventional dispersion only $5.2 \%$ [13].

\section{Parenteral drug delivery}

Nanotechnology is also used in the parenteral drug delivery system (fig. 4). The advantage of this method is it need only less amount of toxic co-solvent for poorly soluble drugs. This will uplift the therapeutic effect of the drug compared with the conventional oral formulation and targeting the drug to the macrophages. The drug clofazimine is given as iv, the concentration in the liver, spleen and lungs reached a high level i.e.; greater than minimum inhibitory concentration, for most of the mycobacterium avium strains. Tarazepide is formulated as nanosuspension in order to overcome the use of surfactants and cyclodextrins to improve the bioavailability [14].

\section{Pulmonary drug delivery}

In pulmonary drug delivery, we are using nano preparations for the drugs which have poor solubility in pulmonary secretions (fig. 5). For the lung delivery, it is nebulized by mechanical or ultrasonic nebulizer. Uniform distribution of the drug is possible and each droplet contains at least single drug particle. Nanosizing improves the diffusion and dissolution of the drug. It enhances the adhesiveness of drug to the mucosal surface and increased residence time at the site of absorption. Nanosuspensions has a fast onset of action initially and then controlled the release of active moiety occur which is required for most of the pulmonary nanosuspensions has a fast onset of action initially and then controlled the release of active moiety occur which is required for most of the pulmonary disease [15]. E. g.; budesonide
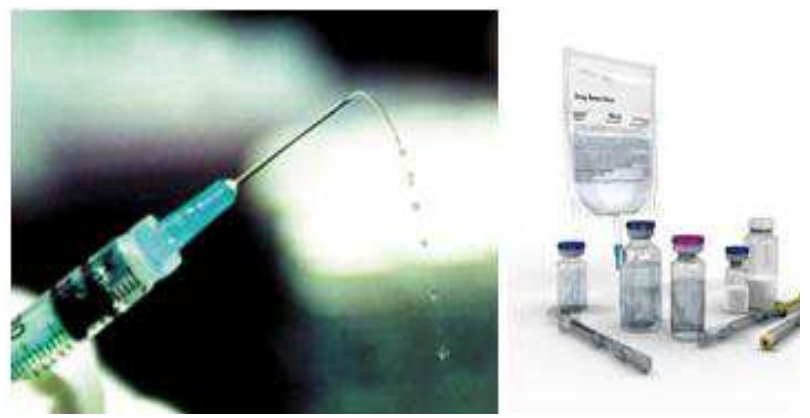

Fig. 5: Nanosuspension for parenteral drug delivery

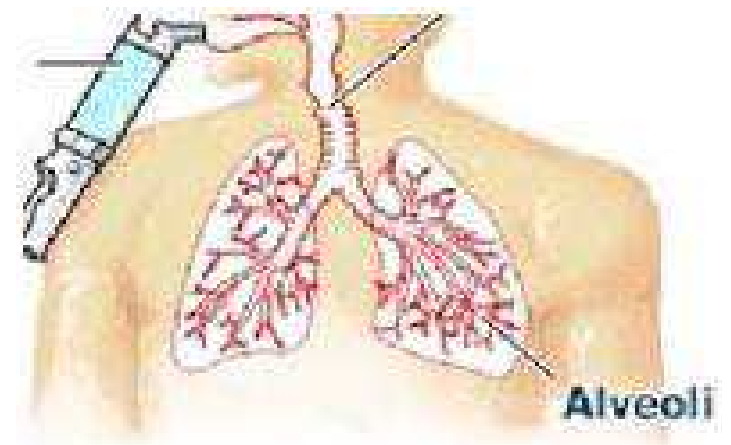

Fig. 6: Nanosuspension for pulmonary drug delivery 


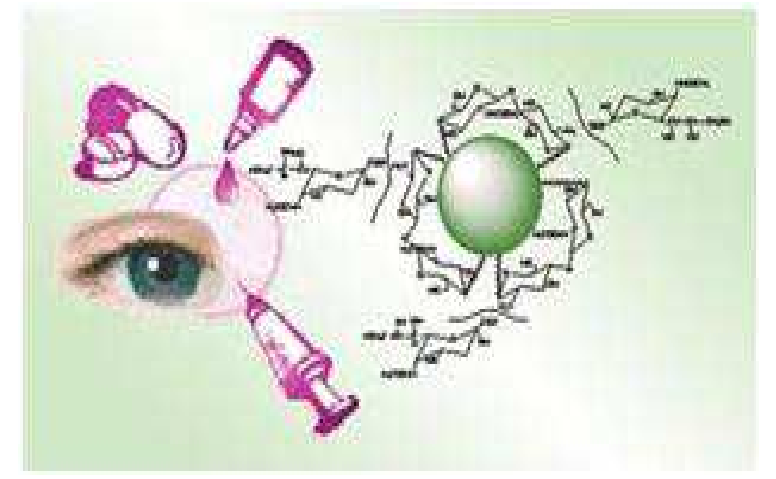

Fig. 7: Nanosuspension for ocular drug delivery

\section{Ocular drug delivery}

Certain drugs have poor solubility in the lachrymal fluid. If it is formulated as nanoparticles its saturation solubility and bioavailability will increase. Mainly applied for hydrophobic drugs. It increases the residence time in cul de sac. The best example of nanosuspension is ibuprofen. The anti-inflammatory activity of ibuprofen increased compared with the aqueous preparation [16].

\section{Targeted drug delivery}

Nanosuspensions also used for targeting their surface properties and changing of the stabiliser can easily alter the in vivo behaviour. The drug will be up taken by the mononuclear phagocytic system to allow regional specific drug delivery. This can be used for targeting antimycobacterial, fungal drugs to the macrophages. Atovaquone is used as targeting nanosuspension to the brain [17].

\section{Mucoadhesion of nanoparticle}

If the nanosuspension is orally administered it diffuse into the liquid medium and adhere to the mucosal surface before absorption. it improves the bioavailability and targeting to the parasite persisting the git. eg; buparvaquone against cryptosporidium parvum [18].

\section{CONCLUSION}

Nanosuspension is a commercially possible approach to solving the poor solubility as well as poor bioavailability problems of the drugs. Productions techniques such as media milling and high-pressure homogenizer are used for large scale production of nano-suspensions. Nanosuspensions can be administered through oral, parenteral, pulmonary, ocular and topical routes. A nanosuspension not only improves the solubility and bioavailability but also modifies the pharmacokinetics of drug and thus improves drug safety and efficacy.

\section{CONFLICT OF INTERESTS}

Declare none

\section{REFERENCES}

1. Lenhardt T, Vergnault G, Grenier P, Scherer D, Langguth P. Evaluation of nanosuspension for absorption enhancement of poorly soluble drugs: in-vitro transport studies across intestinal epithelial monolayers. AAPS J 2008;10:435-8.

2. Arunkumar N, Deecarman M, Rani C. Nanosuspension technology and its application in drug delivery. Asian J Pharm 2009;3:168-73.

3. Xiaohui $\mathrm{Pu}$, Jin Sun, Mol Li, Zhonggui He. Formulation of nanosuspensions as a new approach for the delivery of poorly, current nanoscience. Bentham Sci Publishers Ltd 2009;5:417-27.

4. Liversidge GG, Cundy KC. Particle size reduction for improvement of oral bioavailability of hydrophobic drugs: absolute oral bioavailability of nano-crystalline danazoline beagle dog. Int J Pharm 1995;125:91-7.

5. Shid RL, Dhole SN, Kulkarni N, Shid SL. Nanosuspension: a review. Int J Pharm Sci Rev Res 2013;22:98-106.

6. Senthil Kumar C, VedhaHari BN, Sharavanan SP, Subramanian N, Punitha S, Senthil Kumar V. Novel metronidazole nanosuspension as a controlled drug delivery system for anthelmintic activity. J Pharm Res 2010;3:2404-7.

7. Muller RH, Jacobs C, Kayser O. Nanosuspensions as particulate drug formulations in drug therapy. Rationale for development and what we can expect for the future. Adv Drug Delivery Rev 2001;47:3-19.

8. Kreuter K. Peroral administration of nanoparticles. Adv Drug Delivery Rev 1991;7:71-86.

9. Kavitha VB, Neethu CS, Dineshkumar B, Krishnakumar K, Anish John. Nanosuspension formulation: an improved drug delivery system. Nanoscience and Nanotechnology: An International Journal 2014;4:1-5.

10. Shid RL, Dhole SN, Kulkarni N, Shid SL. Nanosuspension: a review. Int J Pharm Sci Rev 2013;22:98-106.

11. Vaneerdenbrugh B, Vandenmooter G, Augustijns P. Top-down production of drug nanocrystals: nanosuspension stabilization, miniaturization and transformation into solid products. Int J Pharm 2008;364:64-75.

12. Mounica Velmula, Prathibha Pavuluri, S Rajashekar, V Uma Maheswara Rao. Nanosuspension technology for poorly soluble drugs-a review. World J Pharm Pharm Sci 2015;4;1612-25.

13. Peters K, Leitzke S, Diederichs JE, Borner K, Hahn H, Moller RH. Preparation of clofazimine nanosuspensions for intravenous use and evaluation of its therapeutic efficacy in murine mycobacterium avium infection. J Antimicrobe Chemother 2000;45:77-83.

14. Jacobs C, Kayder O, Muller RH. Nanosuspension as a new approach for the formulation of poorly soluble drug tarazepide. Int J Pharma 2000,196:161-4.

15. Kayser 0. Nanosuspension for the formulation of aphidicolin to improve drug targeting effects against Leishmania infected macrophages. Int J Pharma 2000;196:253-6.

16. Ponchel G, Montisci MJ, Dembri A, Durrer C, Duchkne D. Mucoadhesion of colloidal particulate systems in the gastrointestinal tract. Eur J Pharm Biopharm 1997;44:25-31.

17. Chen Y, Liu J, Yang X, Zhao X, Xu H. Oleanolic acid suspension: preparation, in vitro characterization and enhanced hepatoprotective effect. J Pharma Pharmacol 2005;57:259-64.

18. Arunkumar N, Deecaraman M, Rini C. Nano-suspensiontechnology and its applications in drug delivery. Asian J Pharm 2009;3:168-73.

\section{How to cite this article}

- $\quad$ Smita S Aher, Sagar T Malsane, RB Saudagar. Nanosuspension: an overview. Int J Curr Pharm Res 2017;9(3):19-23. 